\title{
POLYNOMIAL APPROXIMATION, LOCAL POLYNOMIAL CONVEXITY, AND DEGENERATE CR SINGULARITIES - II
}

\author{
GAUTAM BHARALI
}

\begin{abstract}
We provide some conditions for the graph of a Hölder-continuous function on $\bar{D}$, where $\bar{D}$ is a closed disc in $\mathbb{C}$, to be polynomially convex. Almost all sufficient conditions known to date - provided the function (say $F$ ) is smooth - arise from versions of the Weierstrass Approximation Theorem on $\bar{D}$. These conditions often fail to yield any conclusion if $\operatorname{rank}_{\mathbb{R}} D F$ is not maximal on a sufficiently large subset of $\bar{D}$. We bypass this difficulty by introducing a technique that relies on the interplay of certain plurisubharmonic functions. This technique also allows us to make some observations on the polynomial hull of a graph in $\mathbb{C}^{2}$ at an isolated complex tangency.
\end{abstract}

\section{INTRODUCTION AND STATEMENT OF RESUlts}

This paper has evolved from the following two considerations:

- Let $\bar{D}$ be a closed disc in $\mathbb{C}$ and let $F \in \mathcal{C}(\bar{D})$. There are numerous results that provide sufficient conditions for the uniform algebra on $\bar{D}$ generated by $z$ and $F$ to equal $\mathcal{C}(\bar{D})$; see [21, 19, 16, 9, 17, 3]. These conditions are sufficient, naturally, for the graph of $F$ to be polynomially convex. The aforementioned results require - either by explicit fiat or through some a priori condition on $F$ - that $F^{-1}\{F(\zeta)\}$ be at most countable for a.e. $\zeta \in \bar{D}$. This is troublesome because it excludes, for instance, $\mathbb{C}\left(\cong \mathbb{R}^{2}\right)$-valued functions in $\mathcal{C}^{1}(\bar{D})$ having $\operatorname{rank}_{\mathbb{R}} D F<2$ on a non-empty open subset of $\bar{D}$. It would thus be useful to devise techniques that allow us to detect polynomial convexity without imposing such restrictions.

- In a recent work, Dieu \& Chi 8] employed a technique that can be applied to situations very different from the one that they study. Their idea, suitably adapted to the given context, might serve as quite a general tool in the study of polynomial convexity of graphs. We wish to argue this case by presenting a couple of adaptations of their idea.

Given $F$ as above and a set $S \subseteq \operatorname{Dom}(F)$, we shall write $\Gamma_{S}(F):=\operatorname{Graph}(F) \cap(S \times \mathbb{C})$. Let us understand some of the known sufficient conditions on $F$, for $\Gamma_{\bar{D}}(F)$ to be polynomially convex, by examining critically a representative result selected from the aforementioned papers. Hence consider:

Result 1.1 ([3], Theorem 1.1). Let $F$ be a complex-valued continuous function on a closed disc $\bar{D} \Subset \mathbb{C}$. Suppose that there is a set $E \subset \bar{D}$ having zero Lebesgue measure such that

- $F^{-1}\{F(\zeta)\}$ is at most countable $\forall \zeta \in \bar{D} \backslash E$;

- For each $\zeta \in \bar{D} \backslash E$, there exists an open sector $S_{\zeta} \varsubsetneqq \mathbb{C}$ with vertex at 0 such that

$$
(z-\zeta)(F(z)-F(\zeta)) \in S_{\zeta} \quad \forall z \in \bar{D} \backslash F^{-1}\{F(\zeta)\} .
$$

2000 Mathematics Subject Classification. Primary 30E10, 32E20, 32F05.

Key words and phrases. Complex tangency, CR singularity, plurisubharmonic functions, polynomially convex, uniform approximation.

This work is supported by the DST via the Fast Track grant SR/FTP/MS-12/2007 and by the UGC under DSA-SAP, Phase IV. 
Then, $[z, F]_{\bar{D}}=\mathcal{C}(\bar{D})$. In particular, $\Gamma_{\bar{D}}(F)$ is polynomially convex.

To clarify before we proceed further: $[z, F]_{\bar{D}}$ denotes the uniform algebra on $\bar{D}$ generated by $z$ and $F$.

Result 1.1 is fairly representative of the key results in many of the papers cited above. It is proved using ideas very similar to those in [21, 19, 16. Observe:

I) the requirement that $F^{-1}\{F(\zeta)\}$ be countable for a.e. $\zeta \in \bar{D}$, and recall the difficulties that it represents. Some version of this condition appears in [21, 19, 16], and is essential to ensuring that the Cauchy transform of any measure $\mu \in \mathcal{C}(\bar{D})^{\star}$ that annihilates $[z, F]_{\bar{D}}$ vanishes a.e. The approximation result then follows from an argument in [4, Theorem 4]. This strategy was first used in [21].

II) that the truth value of the hypothesis of Result 1.1 is, in general, not preserved when $F$ is replaced by $\widetilde{\psi_{F}}$, where $\widetilde{\psi_{F}}$ is that function of which $\psi\left(\Gamma_{\bar{D}}(F)\right)$ is the graph, for a given $\psi \in \operatorname{Aut}\left(\mathbb{C}^{2}\right)$ that preserves $\bar{D} \times \mathbb{C}$. In contrast, polynomial convexity (or the lack thereof) is preserved under such a $\psi$. In fact, functoriality is not an a priori consideration in the proofs of any of the results cited above.

It would be desirable to formulate a theorem that addresses the two problems presented above. A good starting point is to work with an $F$ that has greater regularity than in Result 1.1 With this assumption, ideas that are quite different from the one (sketchily) outlined in (I) become usable. Before we can state our first result, we need:

Definition 1.2. A subset $S \subset \mathbb{C}$ is said to be finitely connected if $\widehat{\mathbb{C}} \backslash S$ has finitely many connected components $(\widehat{\mathbb{C}}$ denotes the one-point compactification of $\mathbb{C})$.

Some notation: given a compact set $K \Subset \mathbb{C}$, we define the class $\mathcal{O}(K)$ as

$$
\begin{aligned}
\mathcal{O}(K):= & \text { the set of functions that are holomorphic on (not necessarily fixed) } \\
& \text { open neighbourhoods of } K .
\end{aligned}
$$

The sub-class $\mathcal{O}_{\zeta}(\bar{D})$ in the following result is the set of functions in $\mathcal{O}(\bar{D})$ that vanish at $\zeta$ (where $\zeta \in \bar{D})$.

Theorem 1.3. Let $F$ be a complex-valued function of Hölder class $\mathcal{C}^{\alpha}(\bar{D}), 0<\alpha<1$, where $\bar{D} \Subset \mathbb{C}$ is a closed disc with centre at 0 . Assume that we can find a nowhere-dense subset $E \subset \bar{D}$, a nowhere-vanishing function $A \in \mathcal{O}(\bar{D})$, positive constants $M, K>0$, and a number $\nu \geq 1$ such that for each $\zeta \in \bar{D} \backslash E$, there exist:

- a function $G_{\zeta} \in \mathcal{O}_{\zeta}(\bar{D})$ satisfying $|z-\zeta|^{-1}\left|G_{\zeta}(z)\right| \leq M \forall z \in \bar{D}$, and

- a constant $C_{\zeta} \in S^{1}$,

so that

$$
C_{\zeta}(z-\zeta)\left(A \cdot F(z)-A \cdot F(\zeta)+G_{\zeta}(z)\right) \in\left\{u+i v \in \mathbb{C}: u \geq 0,|v| \leq K u^{1 / \nu}\right\} \forall z \in \bar{D} .
$$

Then, $\Gamma_{\bar{D}}(F)$ is polynomially convex. If, additionally, $F \in \mathcal{C}^{1}(\bar{D})$ and the set $\left\{z \in \bar{D}: \partial_{\bar{z}} f(z)=0\right\}$ is nowhere dense and finitely connected, then $[z, F]_{\bar{D}}=\mathcal{C}(\bar{D})$.

Lest the profusion of functions make Theorem 1.3 seem very technical, we present the following special case of Theorem 1.3 that has a more concise statement. Concerning the more general statement: the reader is directed to the remark that follows our next result. 
Proposition 1.4. Let $F$ be a complex-valued function of Hölder class $\mathcal{C}^{\alpha}(\bar{D}), 0<\alpha<1$, where $\bar{D} \Subset \mathbb{C}$ is a closed disc with centre at 0 . Assume that we can find a nowhere-dense subset $E \subset \bar{D}$ and constants $K>0, \nu \geq 1$ such that for each $\zeta \in \bar{D} \backslash E, \exists C_{\zeta} \in S^{1}$ so that

$$
C_{\zeta}(z-\zeta)(F(z)-F(\zeta)) \in\left\{u+i v \in \mathbb{C}: u \geq 0,|v| \leq K u^{1 / \nu}\right\} \forall z \in \bar{D} .
$$

Then, $\Gamma_{\bar{D}}(F)$ is polynomially convex. If, additionally, $F \in \mathcal{C}^{1}(\bar{D})$ and the set $\left\{z \in \bar{D}: \partial_{\bar{z}} f(z)=0\right\}$ is nowhere dense and finitely connected, then $[z, F]_{\bar{D}}=\mathcal{C}(\bar{D})$.

Remark 1.5. The function $A \in \mathcal{O}(\bar{D})$ and the functions $G_{\zeta} \in \mathcal{O}_{\zeta}(\bar{D}), \zeta \in \bar{D} \backslash E$, make condition (1.1) more permissive than (1.2). In fact, the hypothesis of Theorem 1.3 is permissive enough to allow us to recover the well-known fact that if $F \in \mathcal{O}(\bar{D})$, then $\Gamma_{\bar{D}}(F)$ is polynomially convex. This will not follow from the more restrictive (1.2), or from any of the sufficient conditions provided by the results cited above. (Of course, the aim of those earlier results was to establish that $[z, F]_{\bar{D}}=\mathcal{C}(\bar{D})$, with polynomial convexity being a by-product.) While these are the intuitions that led to condition (1.1), we also find that this condition is functorial - in the sense of the discussion in (II) above - with respect to any $\psi \in \operatorname{Aut}\left(\mathbb{C}^{2}\right)$ that preserves $\bar{D} \times \mathbb{C}^{2}$. This is demonstrated in the final section of this paper.

Remark 1.6. The interested reader is referred to [15], in which a theorem on the polynomial convexity of $\Gamma_{\bar{D}}(F)$, for $F \in \operatorname{Lip}(1, \bar{D})$, is proved. The ideas central to [15] are different from those associated with the results cited above. Of interest, however, is a nice survey in [15, Section 3] of some known sufficient conditions for polynomial convexity.

The primary tool for proving Theorem 1.3 is the following proposition.

Proposition 1.7. Let $F \in \mathcal{C}(\bar{D})$, where $\bar{D} \Subset \mathbb{C}$ is a closed disc with centre at 0 , and let $\zeta \in \bar{D}$. Suppose there exist a constant $p \geq 2$, a nowhere vanishing function $A \in \mathcal{O}(\bar{D})$, and functions $G, H \in \mathcal{O}_{\zeta}(\bar{D})$ such that

$$
|A \cdot F(z)-A \cdot F(\zeta)+G(z)|^{p} \leq \operatorname{Re}[H(z)(A \cdot F(z)-A \cdot F(\zeta)+G(z))] \forall z \in \bar{D} .
$$

Then

$$
\widehat{\Gamma_{\bar{D}}(F)} \subset\left\{(z, w) \in \bar{D} \times \mathbb{C}:|A(z) w-A \cdot F(\zeta)+G(z)| \leq|H(z)|^{1 /(p-1)}\right\} .
$$

A note on our notation: given a compact $K \Subset \mathbb{C}^{n}, \widehat{K}$ denotes the polynomially convex hull of $K$. The idea behind Proposition 1.7 is taken from a step in the proof of Theorem 2.1 in Dieu \& Chi's paper [8]. However, their idea is not quite in a form that we can directly use. Hence, we provide a complete proof of Proposition 1.7 in the next section.

It also turns out that, when limited to deducing polynomial convexity, Theorem 1.3 subsumes Wermer's theorem in [21. This is discussed in Section 5. In order motivate our next result, it will be helpful to state the result of Wermer that we have referred to several times already.

Result 1.8 (Wermer, [21]). Let $\bar{D} \Subset \mathbb{C}$ be a closed disc. If $F(z)=\bar{z}+R(z)$ on $\bar{D}$ and $R$ satisfies

$$
|R(z)-R(\zeta)|<|z-\zeta|
$$

for all $\zeta, z$ in $\bar{D}$ with $\zeta \neq z$, then $[z, F]_{\bar{D}}=\mathcal{C}(\bar{D})$. In particular, $\Gamma_{\bar{D}}(F)$ is polynomially convex.

One could ask whether we can still infer polynomial convexity if we replace $\bar{z}$ by $\bar{z}^{m}$, where $m \in \mathbb{Z}_{+}$, in Result 1.8 (provided $0 \in \bar{D}$ ). The answer to this question (with one essential amendment) as Theorem [1.9] will show - is, "Yes." Note that if $0 \in \bar{D}, m \geq 2$, and $G$ is differentiable at 0 , then the origin is a point of complex tangency of $\Gamma_{\bar{D}}(F)$; this is what makes our question a non-trivial one. Given a smooth real surface $\mathcal{S} \subset \mathbb{C}^{2}$ and a point $p \in \mathcal{S}$ at which $T_{p}(\mathcal{S})$ is a 
complex line, deciding whether or not $\mathcal{S}$ is locally polynomially convex at $p$ is a subtle problem. We will abbreviate the phrase "point of complex tangency" to $C R$ singularity. When $\mathcal{S}$ has an isolated CR singularity at $p \in \mathcal{S}$ and the order of contact of $T_{p}(\mathcal{S})$ with $\mathcal{S}$ at $p$ equals 2 , we now have a nearly complete understanding of the local polynomial hull of $\mathcal{S}$ at $p$. This knowledge stems from the works of Bishop [5], Forstnerič-Stout [10, and Jöricke [12. Much less is known when the order of contact of $T_{p}(\mathcal{S})$ with $\mathcal{S}$ at an isolated CR singularity $p$ is greater than 2 . Note that when $p$ is a CR singularity, there is a complex-affine change of coordinate centered at $p$ with respect to which $(\mathcal{S}, p)$ is locally a graph. It would be the graph of a function of the form

$$
F(z)=\sum_{j=0}^{m} C_{j} z^{m-j} \bar{z}^{j}+\mathcal{R}(z),
$$

where $\mathcal{R}(z)=O\left(|z|^{m+1}\right)$, if the order of contact of $T_{p}(\mathcal{S})$ with $\mathcal{S}$ at $p$ is $m, m \in \mathbb{Z}_{+}$. In [2, Theorem 1.1], some sufficient conditions were given for $(\mathcal{S}, p)$ to be locally polynomially convex at $p$, provided $C_{m} \neq 0$ in the associated $F$ given by (1.4). These are, however, very technical conditions, and it would be of interest to see whether alternative conditions could be obtained with considerably less technical exertion. Such conditions can be derived from the following

Theorem 1.9. Let $\bar{D} \Subset \mathbb{C}$ be a closed disc with centre at 0 . If $F(z)=\bar{z}^{m}+R(z), z \in \bar{D}$, where $m \in \mathbb{Z}_{+}$, and $R$ satisfies

$$
|R(z)-R(\zeta)|<\left|z^{m}-\zeta^{m}\right| \text { for all } z, \zeta \in \bar{D}: z^{m} \neq \zeta^{m},
$$

then $\Gamma_{\bar{D}}(F)$ is polynomially convex.

Additionally, we can conclude that $[z, F]_{\bar{D}}=\mathcal{C}(\bar{D})$ in the following cases:

- whenever $m=1$ (with no conditions beyond (1.5) on $R$ );

- if $m \geq 2, R \in \mathcal{C}^{1}(\bar{D})$, and $\exists \alpha \in(0,1)$ such that $R$ satisfies the stronger estimate:

$$
|R(z)-R(\zeta)|<\alpha\left|z^{m}-\zeta^{m}\right| \text { for all } z, \zeta \in \bar{D}: z^{m} \neq \zeta^{m} .
$$

Note the various similarities of the above theorem with Result 1.8. However, there does not seem to be any obvious way in which the technique pioneered by Wermer in 21 — i.e. the ideas outlined in (I) above - can be made to work when $m \geq 2$ in Theorem 1.9. It is Proposition 1.7 that provides the key ingredient in its proof. This proof will be presented in Section 4 . We should also mention here De Paepe's generalisation 7 ] of Minsker's Theorem [14. While the concerns of Theorem 1.9 are quite different - e.g., the main result in [7] is a local result — both results involve dealing with functions of a certain pattern that vanish to higher order at $0 \in \mathbb{C}$.

\section{THE KEY PROPOSITION}

Proposition 1.7 is the key proposition on which this paper depends. We devote this section to its proof. That proof, in turn relies on two propositions - one by Hörmander and the other by Catlin - that have been known for a long time, and on a recent result of Poletsky. We begin by stating this result.

Result 2.1 (Poletsky, 18). Let $K$ be a compact subset in an open set $V \subseteq \mathbb{C}^{n}$ and assume that there is a continuous plurisubharmonic function $u$ on $V$ such that $u=0$ on $K$ and positive on $V \backslash K$. If $v$ is a plurisubharmonic function defined on a neighbourhood $W \subset V$ of $K$ and bounded below on $K$, then there exists a plurisubharmonic function $v^{\prime}$ on $V$ that coincides with $v$ on $K$.

With this, we are in a position to provide the: 
Proof of Proposition 1.7 . Let $\Delta$ be an open disc in $\mathbb{C}$ such that $\bar{D} \Subset \Delta$ and $A, G, H \in \mathcal{O}(\Delta)$. Define $\psi: \Delta \times \mathbb{C} \longrightarrow \mathbb{R}$ by

$$
\begin{aligned}
& \psi(z, w):=|A(z) w-A(\zeta) F(\zeta)+G(z)|^{p}-\operatorname{Re}[H(z)(A(z) w-A(\zeta) F(\zeta)+G(z))] \\
& \forall(z, w) \in \Delta \times \mathbb{C},
\end{aligned}
$$

where $\zeta$ and $p$ are as given in the statement of Proposition 1.7. Clearly, $\psi \in \operatorname{psh}(\Delta \times \mathbb{C})$. Let us set $\Gamma:=\Gamma_{\bar{D}}(F)$. Then, $\widehat{\Gamma}$ is polynomially convex. Hence, it follows from a well-known construction by Catlin [6] (see [20, Proposition 1.3] also) that $\exists u \in \operatorname{psh}\left(\mathbb{C}^{2}\right) \cap \mathcal{C}\left(\mathbb{C}^{2}\right)$ such that $u=0$ on $\widehat{\Gamma}$ and $u>0$ on $\mathbb{C}^{2} \backslash \widehat{\Gamma}$.

Now note that, as $\widehat{\Gamma} \Subset \bar{D} \times \mathbb{C}, \psi$ is defined on a neighbourhood of $\widehat{\Gamma}$ and is bounded below on $\widehat{\Gamma}$. Thus, all the conditions in the hypothesis of Result 2.1 are satisfied (taking $K$ to be $\widehat{\Gamma}$ and $v$ to be $\psi$ ). Thus, $\exists \Psi \in \operatorname{psh}\left(\mathbb{C}^{2}\right) \cap \mathcal{C}\left(\mathbb{C}^{2}\right)$ such that

$$
\left.\Psi\right|_{\widehat{\Gamma}}=\left.\psi\right|_{\widehat{\Gamma}} .
$$

We now invoke a result of Hörmander [11, Theorem 4.3.4] that provides an alternative characterisation of polynomial convexity, owing to which:

$$
\widehat{\Gamma}=\left\{(z, w) \in \mathbb{C}^{2}: U(z, w) \leq \sup _{\Gamma} U \forall U \in \operatorname{psh}\left(\mathbb{C}^{2}\right)\right\} .
$$

From this fact and (2.1), it follows that

$$
\Psi(z, w) \leq \sup _{x \in \Gamma} \Psi(x)=\sup _{x \in \Gamma} \psi(x) \leq 0 \forall(z, w) \in \widehat{\Gamma} .
$$

The last inequality is a consequence of the fact that, by the inequality (1.3), $\psi(z, F(z)) \leq 0 \forall z \in \bar{D}$. In other words, owing to (2.1)

$$
\begin{aligned}
|A(z) w-A(\zeta) F(\zeta)+G(z)|^{p} & \leq \operatorname{Re}[H(z)(A(z) w-A(\zeta) F(\zeta)+G(z))] \\
\Longrightarrow|A(z) w-A(\zeta) F(\zeta)+G(z)|^{p} & \leq|H(z)||A(z) w-A(\zeta) F(\zeta)+G(z)| \forall(z, w) \in \widehat{\Gamma} .
\end{aligned}
$$

Therefore, we conclude

$$
\widehat{\Gamma} \subset\left\{(z, w) \in \bar{D} \times \mathbb{C}:|A(z) w-A(\zeta) F(\zeta)+G(z)| \leq|H(z)|^{1 /(p-1)}\right\} .
$$

\section{The Proof of Theorem 1.3}

We will need the version of Mergelyan's theorem given below in order to prove the second part of Theorem 1.3. This version follows easily from Mergelyan's Approximation Theorem [13] - see, for instance, Andersson's observation [1, Remark 6.4].

Result 3.1 (Mergelyan). Suppose $K \subset \mathbb{C}$ is compact and $\widehat{\mathbb{C}} \backslash K$ has a finite number (say $N$ ) of components. Choose one point, say $a_{j}$, from each component. Then, any $f \in \mathcal{C}(K) \cap \mathcal{O}(\operatorname{int}(K))$ can be uniformly approximated by rational functions with poles only at the points $a_{j}, j=1, \ldots, N$.

Here $\widehat{\mathbb{C}}$ denotes the one-point compactification of $\mathbb{C}$.

Proof of Theorem 1.3. Consider a point $\zeta \in \bar{D} \backslash E$ and let $\Delta_{\zeta}$ be an open disc in $\mathbb{C}$ such that $\bar{D} \Subset \Delta_{\zeta}$ and $A, G_{\zeta} \in \mathcal{O}(\Delta)$. Set

$$
\begin{aligned}
H_{\zeta}(z) & :=C_{\zeta}(z-\zeta) \\
\Lambda_{\zeta}(z) & :=H_{\zeta}(z)\left(A \cdot F(z)-A \cdot F(\zeta)+G_{\zeta}(z)\right), z \in \Delta_{\zeta} .
\end{aligned}
$$


Owing to the estimate on $G_{\zeta}, \exists \lambda>1$ such that

$$
\sup _{z \in \bar{D}}\left|(z-\zeta)\left(A \cdot F(z)-A \cdot F(\zeta)+G_{\zeta}(z)\right)\right|<\lambda \forall \zeta \in \bar{D} \backslash E .
$$

Then, writing $p:=\nu(1+(1 / \alpha))$, we estimate

$$
\begin{aligned}
& \frac{\left|A . F(z)-A \cdot F(\zeta)+G_{\zeta}(z)\right|^{p}}{\left(K^{\nu} \lambda+1\right) \operatorname{Re}\left[\Lambda_{\zeta}(z) / \lambda\right]} \\
& \leq\left|A \cdot F(z)-A \cdot F(\zeta)+G_{\zeta}(z)\right|^{p} \\
& \quad \times\left[\lambda^{-\nu}\left[\operatorname{Re}\left(\Lambda_{\zeta}(z)\right)\right]^{\nu}+\left|\operatorname{Im}\left(\Lambda_{\zeta}(z)\right)\right|^{\nu}+\left(K^{\nu} \operatorname{Re}\left(\Lambda_{\zeta}(z)\right)-\left|\operatorname{Im}\left(\Lambda_{\zeta}(z)\right)\right|^{\nu}\right)\right]^{-1} \\
& \leq \lambda^{\nu} \frac{\left|A \cdot F(z)-A \cdot F(\zeta)+G_{\zeta}(z)\right|^{p}}{\left[\operatorname{Re}\left(\Lambda_{\zeta}(z)\right)\right]^{\nu}+\left|\operatorname{lm}\left(\Lambda_{\zeta}(z)\right)\right|^{\nu}} \\
& \quad \leq B \lambda^{\nu} \frac{\left|A \cdot F(z)-A \cdot F(\zeta)+G_{\zeta}(z)\right|^{\nu / \alpha}}{|z-\zeta|^{\nu}} \quad \forall z \in \bar{D} \backslash\left(A \cdot F+G_{\zeta}-A \cdot F(\zeta)\right)^{-1}\{0\} .
\end{aligned}
$$

In the above estimate, the expression $\left[\operatorname{Re}\left(\Lambda_{\zeta}(z)\right)\right]^{\nu}$ makes sense because, by (1.1), $\operatorname{Re}\left(\Lambda_{\zeta}(z)\right) \geq$ $0 \forall z \in \bar{D}$, and the second inequality is again a consequence of (1.1). Here, $B$ denotes a uniform positive constant. In the following steps $B$ will denote a positive constant that is independent of $z$ and $\zeta$, but whose specific value changes from line to line.

Now note that as $A$ is defined on an open neighbourhood of $\bar{D}, A . F \in \mathcal{C}^{\alpha}(\bar{D})$. Combining this fact with the above estimate gives us

$$
\begin{aligned}
\frac{\left|A \cdot F(z)-A \cdot F(\zeta)+G_{\zeta}(z)\right|^{p}}{\left(K^{\nu} \lambda+1\right) \operatorname{Re}\left[\Lambda_{\zeta}(z) / \lambda\right]} \leq B \lambda^{\nu}\{ & \left.\|A \cdot F\|_{\mathcal{C}^{\alpha}(\bar{D})}^{\nu / \alpha}+\frac{M^{\nu / \alpha}|z-\zeta|^{\nu / \alpha}}{|z-\zeta|^{\nu}}\right\} \\
& \forall z \in \bar{D} \backslash\left(A \cdot F+G_{\zeta}-A \cdot F(\zeta)\right)^{-1}\{0\}, \quad \forall \zeta \in \bar{D} \backslash E .
\end{aligned}
$$

We have just shown that there exists a constant $B \gg 1$, independent of $\zeta \in \bar{D} \backslash E$, such that

$$
\left|A \cdot F(z)-A \cdot F(\zeta)+G_{\zeta}(z)\right|^{p} \leq B \operatorname{Re}\left[H_{\zeta}(z)\left(A \cdot F(z)-A \cdot F(\zeta)+G_{\zeta}(z)\right)\right]
$$

$$
\forall z \in \bar{D}, \quad \forall \zeta \in \bar{D} \backslash E .
$$

Then, Proposition 1.7 gives us

$$
\begin{aligned}
& \widehat{\Gamma_{\bar{D}}(F)} \subset\left\{(z, w) \in \bar{D} \times \mathbb{C}:\left|A(z) w-A(\zeta) F(\zeta)+G_{\zeta}(z)\right| \leq(B|z-\zeta|)^{1 /(p-1)}\right\} \\
& \forall \zeta \in \bar{D} \backslash E .
\end{aligned}
$$

Let us first consider any $\zeta \in \bar{D} \backslash E$. Since $A(\zeta) \neq 0$, (3.1) already tells us that

$$
\widehat{\Gamma_{\bar{D}}(F)} \cap(\{\zeta\} \times \mathbb{C})=\{(\zeta, F(\zeta))\} \quad \forall \zeta \in \bar{D} \backslash E .
$$

Next, consider a $\zeta_{*} \in E$. Since $E$ is nowhere dense, there exists a sequence $\left\{\zeta_{n}\right\}_{n \in \mathbb{N}} \subset \bar{D} \backslash E$ such that $\zeta_{n} \longrightarrow \zeta_{*}$ as $n \rightarrow+\infty$. Let $\left(\zeta_{*}, w_{*}\right)$ denote a point in $\widehat{\Gamma_{\bar{D}}(F)} \cap\left(\left\{\zeta_{*}\right\} \times \mathbb{C}\right)$. Then, applying (3.1) with $\zeta=\zeta_{n}, n \in \mathbb{N}$, we get

$$
\begin{aligned}
& \left|A\left(\zeta_{*}\right)\right|\left|w_{*}-F\left(\zeta_{*}\right)\right|-\left|A\left(\zeta_{*}\right)-A\left(\zeta_{n}\right)\right|\left|F\left(\zeta_{*}\right)\right|-\left|A\left(\zeta_{n}\right)\right|\left|F\left(\zeta_{*}\right)-F\left(\zeta_{n}\right)\right|-\left|G_{\zeta_{n}}\left(\zeta_{*}\right)\right| \\
& \quad \leq\left|A\left(\zeta_{*}\right) w_{*}-A \cdot F\left(\zeta_{n}\right)+G_{\zeta_{n}}\left(\zeta_{*}\right)\right| \\
& \quad \leq\left(B\left|\zeta_{*}-\zeta_{n}\right|\right)^{1 /(p-1)} \forall n \in \mathbb{N} .
\end{aligned}
$$

If we now set

$$
\begin{array}{rlrl}
\mu & :=\inf _{\bar{D}}|A|, & & N_{1}:=\sup _{\bar{D}}|F| \\
N_{2}:=\left\|\left.A\right|_{\bar{D}}\right\|_{\mathcal{C}^{\alpha}(\bar{D})}, & & N_{3}:=\|F\|_{\mathcal{C}^{\alpha}(\bar{D})}
\end{array}
$$


then, we infer that

$$
\left|w_{*}-F\left(\zeta_{*}\right)\right| \leq \frac{\left(B\left|\zeta_{*}-\zeta_{n}\right|\right)^{1 /(p-1)}+\left(N_{1} N_{2}+N_{2} N_{3}\right)\left|\zeta_{*}-\zeta_{n}\right|^{\alpha}+M\left|\zeta_{*}-\zeta_{n}\right|}{\mu}
$$

$\longrightarrow 0$ as $n \rightarrow+\infty$.

Since $\zeta_{*}$ above was arbitrarily picked from $E$, this implies that

$$
\widehat{\Gamma_{\bar{D}}(F)} \cap\left(\left\{\zeta_{*}\right\} \times \mathbb{C}\right)=\left\{\left(\zeta_{*}, F\left(\zeta_{*}\right)\right)\right\} \quad \forall \zeta_{*} \in E .
$$

Since $\widehat{\Gamma_{\bar{D}}(F)} \subset \bar{D} \times \mathbb{C},(3.2)$ and $(3.3)$ tell us that $\widehat{\Gamma_{\bar{D}}(F)}=\Gamma_{\bar{D}}(F)$. This establishes the first part of Theorem 1.3

For any compact set $L \Subset \mathbb{C}$, let $\mathcal{R}(L)$ denote the class of functions on $L$ that are uniformly approximable on $L$ by rational functions whose poles lie outside $L$. Assuming now that $F \in \mathcal{C}^{1}(\bar{D})$, set $L_{F}:=\left\{z \in \bar{D}: \partial_{\bar{z}} F(z)=0\right\}$. In view of our assumptions on the topology of the set $L_{F}$, Result 3.1 implies that

$$
\mathcal{R}\left(L_{F}\right)=\mathcal{C}\left(L_{F}\right) .
$$

At this stage we can invoke a result of Wermer [22, page 9], which says:

Let $F \in \mathcal{C}^{1}(\bar{D})$ and assume that $\Gamma_{\bar{D}}(F)$ is polynomially convex. Then $[z, F]_{\bar{D}}$ consists exactly of those continuous functions on $\bar{D}$ whose restrictions to $L_{F}$ belong to $\mathcal{R}\left(L_{F}\right)$.

We have already established that $\Gamma_{\bar{D}}(F)$ is polynomially convex. Thus, in view of (3.4), we deduce that $[z, F]_{\bar{D}}=\mathcal{C}(\bar{D})$.

\section{The Proof of Theorem 1.9}

We will emulate many of the notations and computations used in the proof of Theorem 1.3 For each $\zeta \in \bar{D}$, let us define

$$
\Lambda_{\zeta}(z):=\left(z^{m}-\zeta^{m}\right)(F(z)-F(\zeta)), z \in \bar{D}
$$

Now observe that

$$
\left|\Lambda_{\zeta}(z)-\right| z^{m}-\left.\zeta^{m}\right|^{2} \mid \begin{cases}\leq\left|z^{m}-\zeta^{m}\right|^{2} & \forall z \in \bar{D}, \\ <\left|z^{m}-\zeta^{m}\right|^{2} & \forall z: z^{m} \neq \zeta^{m} .\end{cases}
$$

This tells us that $\Lambda_{\zeta}(z)$ lies in the disc $D\left(\left|z^{m}-\zeta^{m}\right|^{2} ;\left|z^{m}-\zeta^{m}\right|^{2}\right)$ whenever $z^{m} \neq \zeta^{m}$. Thus, if $r$ denotes the radius of $\bar{D}$, then

$$
\Lambda_{\zeta}(z) \in \overline{D\left(4 r^{2 m} ; 4 r^{2 m}\right)} \forall(z, \zeta) \in \bar{D} \times \bar{D} .
$$

It is elementary to infer from this that

$$
\Lambda_{\zeta}(z) \in\left\{u+i v \in \mathbb{C}: u \geq 0,|v| \leq \sqrt{8} r^{m} u^{1 / 2}\right\} \forall(z, \zeta) \in \bar{D} \times \bar{D} .
$$

Note the resemblance of the above to condition to the condition (1.1) in Theorem 1.3. Hence, just as in the proof of Theorem 1.3, if we pick a $\lambda>1$ such that $\lambda$ satisfies

$$
\sup _{\bar{D}}\left|\Lambda_{\zeta}(z)\right|<\lambda \forall \zeta \in \bar{D}
$$

then a string of estimates analogous to the one in the proof of Theorem 1.3 leads to

$$
\frac{|F(z)-F(\zeta)|^{4}}{\left(8 r^{2 m} \lambda+1\right) \operatorname{Re}\left[\Lambda_{\zeta}(z) / \lambda\right]} \leq \lambda^{2} \frac{|F(z)-F(\zeta)|^{2}}{\left|z^{m}-\zeta^{m}\right|^{2}} \forall z \in \bar{D}: z^{m} \neq \zeta^{m} .
$$


Applying the condition (1.5) to the above estimate, we get

$$
|F(z)-F(\zeta)|^{4} \leq 4 \lambda\left(8 r^{2 m} \lambda+1\right) \operatorname{Re}\left[\left(z^{m}-\zeta^{m}\right)(F(z)-F(\zeta))\right] \quad \forall(z, \zeta) \in \bar{D} \times \bar{D} .
$$

Let us denote the uniform constant on the right-hand side of (4.3) by $B$. Combining this inequality with Proposition 1.7, we get:

$$
\widehat{\Gamma_{\bar{D}}(F)} \subset\left\{(z, w) \in \bar{D} \times \mathbb{C}:|w-F(\zeta)| \leq\left(B\left|z^{m}-\zeta^{m}\right|\right)^{1 / 3}\right\} \quad \forall \zeta \in \bar{D} .
$$

Clearly, $\widehat{\Gamma_{\bar{D}}(F)} \cap(\{\zeta\} \times \mathbb{C})=\{(\zeta, F(\zeta))\} \quad \forall \zeta \in \bar{D}$. We have thus established that $\Gamma_{\bar{D}}(F)$ is polynomially convex.

The second assertion of Theorem 1.9 for the case $m=1$ is just the conclusion of Result 1.8. Let us thus consider the case when $m \geq 2$. Let us fix a $z \neq 0$. Then, we can find a sequence $\left\{r_{n}\right\}_{n \in \mathbb{N}} \subset(\mathbb{R} \backslash\{0\})$ such that

- $\lim _{n \rightarrow \infty} r_{n}=0$;

- $\left(z+r_{n}\right)^{m} \neq z^{m} \forall n \in \mathbb{N}$;

- $\left(z+i r_{n}\right)^{m} \neq z^{m} \forall n \in \mathbb{N}$.

By our assumptions on $R$ :

$$
\frac{\partial F}{\partial \bar{z}}(z)=m \bar{z}^{m-1}+\lim _{n \rightarrow \infty} \frac{1}{2}\left(\frac{R\left(z+r_{n}\right)-R(z)}{r_{n}}-\frac{R\left(z+i r_{n}\right)-R(z)}{i r_{n}}\right) .
$$

Note that, by the properties of the sequence $\left\{r_{n}\right\}_{n \in \mathbb{N}}$, we can find an $N \in \mathbb{Z}_{+}$sufficiently large that:

$$
\begin{aligned}
\frac{\left|R\left(z+r_{n}\right)-R(z)\right|}{\left|r_{n}\right|}<\alpha \frac{\left|\left(z+r_{n}\right)^{m}-z^{m}\right|}{\left|r_{n}\right|} & \leq \alpha \sum_{j=0}^{m-1}\left|z+r_{n}\right|^{j}|z|^{m-1-j} \\
& \leq m \frac{1+\alpha}{2}|z|^{m-1} \forall n \geq N
\end{aligned}
$$

A similar estimate holds for the second difference quotient in (4.5). Combining (4.6) with (4.5), we get

$$
\left|\frac{\partial F}{\partial \bar{z}}(z)\right| \geq m|z|^{m-1}-m \frac{1+\alpha}{2}|z|^{m-1}>0,
$$

provided $z \neq 0$. Hence, in the terminology of the previous section,

$$
L_{F}:=\left\{z \in \bar{D}: \partial_{\bar{z}} F(z)=0\right\}=\{0\} .
$$

We conclude with the same argument as in the final paragraph of the proof of Theorem 1.3. Since we have already established that $\Gamma_{\bar{D}}(F)$ is polynomially convex, and $L_{F}$ is a singleton, 22] implies that $[z, F]_{\bar{D}}=\mathcal{C}(\bar{D})$.

\section{The Relation of Theorem 1.3 to known Results}

This section is dedicated to elaborating upon two observations made in Section 1 
5.1. The invariance of the hypothesis of Theorem 1.3 under the action of certain elements of $\operatorname{Aut}\left(\mathbb{C}^{2}\right)$ : Let $\bar{D}$ and $F$ be as given in Theorem 1.3 . It is a tautology that polynomial convexity of $\Gamma_{\bar{D}}(F)$ (or the lack thereof) is preserved when transformed by any $\psi \in \operatorname{Aut}\left(\mathbb{C}^{2}\right)$ that maps $\bar{D} \times \mathbb{C}$ onto itself. We claim that the truth (or falsity) of the hypothesis of Theorem 1.3 too is preserved when $F$ is replaced by that function of which $\psi\left(\Gamma_{\bar{D}}(F)\right)$ is a graph. To establish this, it suffices to accomplish the following:

- given a $\psi \in \operatorname{Aut}\left(\mathbb{C}^{2}\right)$ that maps $\bar{D} \times \mathbb{C}$ onto itself, show that there is a function (in the notation of (II), Section 10 $\widetilde{\psi} F \in \mathcal{C}^{\alpha}(\bar{D})$ such that $\psi\left(\Gamma_{\bar{D}}(F)\right)=\Gamma_{\bar{D}}(\widetilde{\psi} F)$.

- assuming that $F$ satisfies the hypothesis of Theorem 1.3, produce a nowhere dense $\widetilde{E} \subset \bar{D}$; a constant $\widetilde{M}>0$; a function $\widetilde{A} \in \mathcal{O}(\bar{D})$ that vanishes nowhere on $\bar{D}$; and, associated to each $\zeta \in \bar{D} \backslash \widetilde{E}$, constants $\widetilde{C_{\zeta}} \in S^{1}$ and functions $\widetilde{G_{\zeta}} \in \mathcal{O}_{\zeta}(\bar{D})$ such that the statement obtained by replacing $F$ by $\widetilde{\psi} F$, and the other objects occurring in the hypothesis of Theorem 1.3 by their analogues listed above, is also true.

Let us set

$$
\mathfrak{G}(\bar{D}):=\left\{\psi \in \operatorname{Aut}\left(\mathbb{C}^{2}\right): \psi(\bar{D} \times \mathbb{C})=\bar{D} \times \mathbb{C}\right\}
$$

and determine all the elements of $\mathfrak{G}(\bar{D})$. For a $\psi \in \mathfrak{G}(\bar{D})$, write $\psi=\left(\psi_{1}, \psi_{2}\right)$. For each $z \in \bar{D}$, the functions $\psi_{1}(z, \cdot)$ are entire functions that map $\mathbb{C}$ to $\bar{D} \Subset \mathbb{C}$. Hence, it follows from Liouville's theorem that $\psi_{1}$ depends only on $z$. This implies that $\psi_{1} \in \operatorname{Aut}(\mathbb{C})$ and, as $\psi_{1}(\bar{D})=\bar{D}, \exists \phi \in \mathbb{R}$ such that

$$
\psi_{1}(z, w)=e^{i \phi} z \forall(z, w) \in \mathbb{C}^{2} .
$$

Furthermore, since $\psi$ must be injective, it follows that $\psi_{2}(z, \cdot) \in \operatorname{Aut}(\mathbb{C})$ for each $z \in \mathbb{C}^{2}$. It can now easily be shown that there exist entire functions $\mathcal{G}, \mathcal{H}$, where $\mathcal{G}$ is nowhere-vanishing, such that

$$
\psi \in \mathfrak{G}(\bar{D}) \Longrightarrow \psi(z, w)=\left(e^{i \phi} z, \mathcal{G}(z) w+\mathcal{H}(z)\right) \forall(z, w) \in \mathbb{C}^{2}
$$

It is, of course, obvious that each $\psi$ having the form given in (5.1) belongs to $\mathfrak{G}(\bar{D})$.

Now assume that $F$ satisfies the hypothesis on Theorem 1.3. In view of (5.1), if $\psi \in \mathfrak{G}(\bar{D})$, then

$$
\widetilde{\psi_{F}}(z)=\mathcal{G}\left(e^{-i \phi} z\right) F\left(e^{-i \phi} z\right)+\mathcal{H}\left(e^{-i \phi} z\right) \forall z \in \bar{D},
$$

for some $\phi \in \mathbb{R}$ and $\mathcal{G}, \mathcal{H} \in \mathcal{O}(\mathbb{C})$ such that $\mathcal{G}$ is non-vanishing. Since $\mathcal{G}, \mathcal{H} \in \mathcal{O}(\mathbb{C})$, it is immediate that $\widetilde{\psi_{F}} \in \mathcal{C}^{\alpha}(\bar{D})$.

Let us introduce two notations. Let

$$
\begin{aligned}
\widetilde{E} & :=e^{i \phi} E, \\
\mathcal{W}(K, \nu) & :=\left\{u+i v \in \mathbb{C}: u \geq 0,|v| \leq K u^{1 / \nu}\right\} .
\end{aligned}
$$

Then, for each $\zeta \in \bar{D} \backslash \widetilde{E}$, write

$$
\Lambda_{\zeta}(z):=C_{e^{-i \phi} \zeta} e^{-i \phi}(z-\zeta)\left(A \cdot F\left(e^{-i \phi} z\right)-A \cdot F\left(e^{-i \phi} \zeta\right)+G_{e^{-i \phi} \zeta}\left(e^{-i \phi} z\right)\right),
$$

where $C_{e^{-i \phi} \zeta} \in S^{1}$ and $G_{e^{-i \phi} \zeta} \in \mathcal{O}(\bar{D})$ are exactly as provided by the hypothesis of Theorem 1.3 , this makes sense because $\zeta \in \bar{D} \backslash \widetilde{E}$ in the preceding definition. It is obvious, given our assumption that $F$ satisfies the hypothesis of Theorem 1.3 , that

$$
\Lambda_{\zeta}(z) \in \mathcal{W}(K, \nu) \forall z \in \bar{D} \text { and } \forall \zeta \in \bar{D} \backslash \widetilde{E} .
$$


It is now follows from a simple computation that if we define

$$
\begin{aligned}
\widetilde{A} & :=\frac{A}{\mathcal{G}}\left(e^{-i \phi} \cdot\right), & \widetilde{G_{\zeta}} & :=G_{e^{-i \phi} \zeta}\left(e^{-i \phi} \cdot\right)-\frac{A \cdot \mathcal{H}}{\mathcal{G}}\left(e^{-i \phi} \cdot\right)+\frac{A \cdot \mathcal{H}}{\mathcal{G}}\left(e^{-i \phi} \zeta\right), \\
\widetilde{C_{\zeta}} & :=e^{-i \phi} C_{e^{-i \phi} \zeta} & & \forall \zeta \in \bar{D} \backslash \widetilde{E},
\end{aligned}
$$

then

$$
\Lambda_{\zeta}(z)=\widetilde{C_{\zeta}}(z-\zeta)\left(\widetilde{A} \cdot \widetilde{\psi} F(z)-\widetilde{A} \cdot \widetilde{\psi_{F}}(\zeta)+\widetilde{G_{\zeta}}(z)\right) \forall z \in \bar{D} \text { and } \forall \zeta \in \bar{D} \backslash \widetilde{E} .
$$

From (5.2) and (5.3), it follows that if $F$ satisfies the hypothesis of Theorem 1.3 , then the following statement:

We can find a nowhere-dense subset $\widetilde{E} \subset \bar{D}$, a nowhere vanishing function $\widetilde{A} \in \mathcal{O}(\bar{D})$, positive constants $\widetilde{M}, K>0$, and a number $\nu \geq 1$ such that for each $\zeta \in \bar{D} \backslash \widetilde{E}$, there exist:

- a function $\widetilde{G_{\zeta}} \in \mathcal{O}_{\zeta}(\bar{D})$ satisfying $|z-\zeta|^{-1}\left|\widetilde{G_{\zeta}}(z)\right| \leq \widetilde{M} \forall z \in \bar{D}$, and

- a constant $\widetilde{C}_{\zeta} \in S^{1}$,

so that

$$
\widetilde{C_{\zeta}}(z-\zeta)\left(\widetilde{A} \cdot \widetilde{\psi} F(z)-\widetilde{A} \cdot \widetilde{\psi} F(\zeta)+\widetilde{G_{\zeta}}(z)\right) \in \mathcal{W}(K, \nu) \forall z \in \bar{D} .
$$

also holds true (with $K$ and $\nu$ having exactly the same values as hypothesized in Theorem 1.3). As $\psi$ is invertible, the last implication is sufficient to establish that the hypothesis of Theorem[1.3 is functorial with respect to $\mathfrak{G}(\bar{D})$.

5.2. The relation between Theorem $\mathbf{1 . 3}$ and Wermer's theorem (Result 1.8): We remark that when we consider the weaker form of Wermer's theorem - i.e. taking its conclusion as merely that $\Gamma_{\bar{D}}(F)$ is polynomially convex - then this weaker result is subsumed by Theorem 1.3. To see this, we first observe that, without loss of generality, we may take $\bar{D}$ in Wermer's theorem to be a disc centred at $0 \in \mathbb{C}$. Next, we draw the reader's attention to the argument in the first paragraph of the proof of Theorem 1.9. By this argument, and from the assumptions on $F$ in Wermer's theorem, it follows that

$$
(z-\zeta)(F(z)-F(\zeta)) \in\left\{u+i v \in \mathbb{C}: u \geq 0,|v| \leq \sqrt{8} r u^{1 / 2}\right\}
$$

where $r>0$ is the radius of the disc $\bar{D}$. The above shows that the functions considered by Result 1.8 are a special case of the functions considered by Theorem 1.3 .

Acknowledgement. I thank the anonymous referee of an earlier version of this work for helpful comments on exposition and for drawing my attention to work of which I had been unaware.

\section{REFERENCES}

[1] M. Andersson, Topics in Complex Analysis, Universitext, Springer-Verlag, New York, 1997.

[2] G. Bharali, Surfaces with degenerate CR singularities that are locally polynomially convex, Michigan Math. J. 53 (2005), 429-445.

[3] G. Bharali, Polynomial approximation, local polynomial convexity, and degenerate CR singularities, J. Funct. Anal. 236 (2006), 351-368.

[4] E. Bishop, A minimal boundary for function algebras, Pacific J. Math. 9 (1959), 629-642.

[5] E. Bishop, Differentiable manifolds in complex Euclidean space, Duke Math J. 32 (1965), 1-21.

[6] D. Catlin, Boundary behavior of holomorphic functions on weakly psedoconvex domains, Dissertation, Princeton University, 1978.

[7] P.J. de Paepe, Approximation on disks, Proc. Amer. Math. Soc. 97 (1986), 299-302.

[8] N.Q. Dieu and K.P. Chi, Local polynomial convexity of certain graphs in $\mathbb{C}^{2}$, Michigan Math. J. 58, 479-488.

[9] J. Duval, Un exemple de disque polynômialement convexe, Math. Ann. 281 (1988), 583-588.

[10] F. Forstnerič and E.L. Stout, A new class of polynomially convex sets, Arkiv Mat. 29 (1991), 51-62. 
[11] L. Hörmander, An Introduction to Complex Analysis in Several Variables, 3rd edition (revised), North-Holland, Amsterdam, The Netherlands, 1990.

[12] B. Jöricke, Local polynomial hulls of discs near isolated parabolic points, Indiana Univ. Math. J. 46 (1997), 789-826.

[13] S.N. Mergelyan, Uniform approximations of functions of a complex variable (Russian), Uspekhi Mat. Nauk 7 (48) (1952), 31-122.

[14] S. Minsker, Some applications of the Stone-Weierstrass theorem to planar rational approximation, Proc. Amer. Math. Soc. 58 (1976), 94-96.

[15] A.G. O'Farrell and K.J.Preskenis, Approximation by polynomials in two complex variables, Math. Ann. 246 (1979-80), 225-232.

[16] A.G. O'Farrell and K.J.Preskenis, Approximation by polynomials in two diffeomorphisms, Bull. Amer. Math. Soc. (N.S.) 10 (1984), 105-107.

[17] A.G. O'Farrell and K.J.Preskenis, Uniform approximation by polynomials in two functions, Math. Ann. 284 (1989), 529-535.

[18] E. A. Poletsky, Jensen measures and analytic multifunctions, Ark. Mat. 42 (2004), no. 2, 335-352.

[19] K.J. Preskenis, Approximation by polynomials in $z$ and another function, Proc. Amer. Math. Soc. 68 (1978), 69-74.

[20] N. Sibony, Some aspects of weakly pseudoconvex domains, Several Complex Variables and Complex Geometry, Part 1 (Santa Cruz, CA, 1989), Proc. Sympos. Pure. Math. 52, Part 1, AMS, Providence, RI, 1991.

[21] J. Wermer, Approximation on a disk, Math. Ann. 155 (1964), 331-333.

[22] J. Wermer, Polynomially convex disks, Math. Ann. 158 (1965), 6-10.

Department of Mathematics, Indian Institute of Science, Bangalore 560012, India

E-mail address: bharali@math.iisc.ernet.in 